\title{
Heat and gravitation. II. Stability
}

\author{
Christian Frønsdal
}

Received: 19 May 2010 / Accepted: 11 August 2010 / Published online: 11 September 2010 (C) The Author(s) 2010. This article is published with open access at Springerlink.com

\begin{abstract}
Some features of hydro- and thermodynamics, as applied to atmospheres and to stellar structures, are puzzling. 1. The suggestion, first made by Laplace, that our atmosphere has an adiabatic temperature distribution, is confirmed for the lower layers, but the reason why it should be so is difficult to understand. 2. The standard treatment of relativistic thermodynamics does not allow for a systematic treatment of mixtures, such as the mixture of a perfect gas with radiation. 3. The concept of mass in applications of general relativity to stellar structures is less than completely satisfactory. 4. Arguments in which a concept of energy plays a role, in the context of hydro-thermodynamical systems and gravitation, are not always convincing. It was proposed that a formulation of thermodynamics as an action principle may be a suitable approach to adopt for a new investigation of these matters. In this second article of a series we propose to base criteria of stability on the hamiltonian functional that is provided by the variational principle, to replace the reliance that has often been placed on ad hoc definitions of the "energy". We introduce a new virial principle that is formulated entirely within the Eulerian description of hydrodynamics, which allows a simpler derivation of a well known stability criterion for polytropic stellar configurations. Boundary conditions are based entirely on mass conservation. The new approach is tested on isothermal and polytropic atmospheres and then used to initiate a new study of stars. Traditional results for polytropic, spherical configurations are confirmed, but our study gives new insight and new results for the case that radiation is taken into account.
\end{abstract}

Keywords Polytropes $\cdot$ Stability $\cdot$ Action principle

C. Frønsdal ( $\varangle)$

Physics Department, University of California, Los Angeles, CA 90095-1547, USA

e-mail: fronsdal@physics.ucla.edu 


\section{Introduction}

Stability of a dynamical system that consists of a gas held together by mutual gravitational forces can be studied by comparing the energy of a presumptive bound configuration with that of an infinitely diffuse state. But a necessary condition, for such comparison to carry any weight, is that a framework exists within which a well defined hamiltonian functional of the dynamical variables can be provided.

The literature contains stability studies where this condition, if not clearly stated, was nevertheless satisfied. But there are numerous instances, even in textbooks, where an expression for the energy is written down without any supportive dynamical framework.

In these papers we insist on basing all investigations on a variational principle. The dynamical equations are in most cases the same as those of previous studies, but in the case that the effect of radiation is included we are breaking new ground.

The paper begins with a very brief summary of the first paper of this series [10]. A well known variational principle for irrotational hydrodynamics is expanded by elevating the temperature to the role of an independent dynamical variable (Sect. 2.1). After a brief summary of standard atmospheric models we introduce a new "virial" theorem (Sect. 2.3). It is an analogue of the classical virial theorem, in which the particle coordinate operator $\vec{r} \cdot \vec{p}$ is replaced by $\rho \Phi$, where $\rho$ is a mass density and $\Phi$ is the velocity potential (the canonical conjugate of $\rho$ ). Using this new virial theorem in a manner that recalls Jacobi's original use of the classical analogue we are led to the well known stability criterion $(n<3)$ for polytropic atmospheres. No appeal is made to kinetic theory and no approximations are made.

Section 3 investigates the stability of several atmospheric models. The boundary conditions, that are central to the question of stability, are deduced in each case from the conservation of mass. This is a reminder of the fact that the usual approach to relativistic thermodynamics abandons the continuity equation of classical hydrodynamics, a defect that can easily be repaired [9].

We study the stability of some atmospheric models by perturbation theory. The important role of mass conservation in the choice of initial conditions is emphasized.

This paper is limited to the non relativistic approximation. The relativistic case will be examined in another paper of this series.

\subsection{Hydrodynamics}

Basic hydrodynamics deals with a density field $\rho$ and a velocity field $\vec{v}$ over $\mathbb{R}^{3}$, subject to two fundamental equations, the equation of continuity,

$$
\dot{\rho}+\operatorname{div}(\rho \vec{v})=0, \quad \dot{\rho}:=\frac{\partial \rho}{\partial t},
$$

and the hydrodynamical equation [2]

$$
-\operatorname{grad} p=\rho \frac{D}{D t} \vec{v}:=\rho(\dot{\vec{v}}+\vec{v} \cdot \operatorname{grad} \vec{v}) .
$$

This involves another field, the scalar field $p$, interpreted as the local pressure. 
We assume that the velocity field can be represented as the gradient of a scalar field,

$$
\vec{v}=-\operatorname{grad} \Phi
$$

In this case the hydrodynamical condition is reduced to

$$
\operatorname{grad} p=\rho \operatorname{grad}\left(\dot{\Phi}-\vec{v}^{2} / 2\right)
$$

To complete this system one needs a relation between the fields $p$ and $\rho$.

A local functional $V[\rho]$ is defined up to an additive linear term by

$$
p=\rho V^{\prime}-V, \quad V^{\prime}:=d V / d \rho .
$$

Then $d p=\rho d V^{\prime}$ and Eq. (1.4) becomes, if $\rho \neq 0$,

$$
V^{\prime}=\dot{\Phi}-\vec{v}^{2} / 2+\lambda, \quad \lambda \text { constant }
$$

The two fundamental equations that characterize irrotational hydrodynamics are

$$
\begin{aligned}
& \dot{\rho}+\operatorname{div}(\rho \vec{v})=0, \quad \dot{\rho}:=\partial \rho / \partial t, \\
& \dot{\Phi}-\vec{v}^{2} / 2+\lambda=\partial V / \partial \rho,
\end{aligned}
$$

together with the defining equations $\vec{v}=-\operatorname{grad} \Phi, p:=\rho V^{\prime}-V$. These equations are the Euler-Lagrange equations associated with the action [8]

$$
A[\rho, \Phi]=\int d t d^{3} x \mathcal{L}, \quad \mathcal{L}=\rho\left(\dot{\Phi}-\vec{v}^{2} / 2+\lambda\right)-V[\rho]
$$

The value of this last circumstance in the present context lies in the fact that it gives us a valid concept of a total energy functional.

\subsection{The mass}

The conserved density $\rho$ will be taken to have the interpretation of mass density, and the total mass is the constant of the motion

$$
M=\int d^{3} x \rho
$$

Since the total mass is a constant of the motion it is natural to fix it in advance and to vary the action subject to the constraint $\int d^{3} x \rho(x)=M$. The parameter $\lambda$ takes on the role of a Lagrange multiplier and the action takes the form

$$
A=\int d^{3} x\left(\rho\left(\dot{\Phi}-\vec{v}^{2} / 2-\phi\right)-V\right)+\lambda\left(\int d^{3} x \rho-M\right) .
$$

The gravitational potential $\phi$ is included. 
The conservation of mass has important implications for boundary conditions.

1.3 Isothermal and polytropic atmospheres

An ideal gas at equilibrium, with constant temperature, obeys the gas law

$$
p / \rho=\mathcal{R} T \text {. }
$$

Pressure and density are in cgs units and $\mu \mathcal{R}=0.8314 \times 10^{8} \mathrm{erg} / \mathrm{K}$, where $\mu$ is the atomic weight.

In hydrodynamics, the isothermal atmosphere can be given a lagrangian treatment by taking

$$
V=\mathcal{R} T \rho \log \rho .
$$

We suppose that the gas is confined to the section $z_{0}<z<z_{0}+h$ of a vertical cylinder with base area $\mathcal{A}$ and expect the density to fall off at higher altitudes. The action density, for a gas at constant temperature $T$ in a constant gravitational field $\phi=g z, g$ constant, is

$$
\mathcal{L}[\Phi, \rho]=\rho\left(\dot{\Phi}-\vec{v}^{2} / 2-g z+\lambda\right)-\mathcal{R} T \rho \log \rho
$$

We may consider this an isolated system with fixed mass.

At equilibrium $\dot{\Phi}=0, \vec{v}=0, \dot{\rho}=0$ and the equation of motion is $V^{\prime}=\mathcal{R} T(1+$ $\log \rho)=\lambda-g z$, hence

$\rho(x, y, z)=\mathrm{e}^{-1+\lambda / \mathcal{R} \mathrm{T}} \mathrm{e}^{-\mathrm{gz} / \mathcal{R} \mathrm{T}}, \quad \mathrm{M}=\mathcal{A} \frac{\mathcal{R} \mathrm{T}}{\mathrm{g}} \mathrm{e}^{-1+\lambda / \mathcal{R} \mathrm{T}}\left(1-\mathrm{e}^{-\mathrm{gh} / \mathrm{RT}}\right) \mathrm{e}^{-\mathrm{gz}_{0} / \mathcal{R} \mathrm{T}}$

and after elimination of $\lambda$

$$
\rho=\frac{g M}{\mathcal{A} \mathcal{R} T} \frac{\mathrm{e}^{-\mathrm{g}\left(\mathrm{z}-\mathrm{z}_{0}\right) / \mathcal{R} \mathrm{T}}}{1-\mathrm{e}^{-\mathrm{gh} / \mathrm{RT}}}, \quad p=\frac{g M}{\mathcal{A}} \frac{\mathrm{e}^{-\mathrm{g}\left(\mathrm{z}-\mathrm{z}_{0}\right) / \mathcal{R} \mathrm{T}}}{1-\mathrm{e}^{-\mathrm{gh} / \mathrm{RT}}} .
$$

(If the atmosphere extends to infinity the pressure at the bottom is $g M / \mathcal{A}$, as it should be.) A difficulty with this model is that the specific internal energy is $\mathcal{R} T \log \rho$, which is very different from that of an ideal gas.

The isothermal atmosphere is usually abandoned in favor of the polytropic atmosphere. A polytropic gas can be described by the lagrangian (1.9), with

$$
V=\hat{a} \rho^{\gamma}, \quad \hat{a}, \gamma \text { constant }
$$

Variation with respect to $\rho$ gives

$$
p=\frac{\hat{a}}{n} \rho^{\gamma}, \quad \frac{1}{n}=\gamma-1 .
$$


The temperature does not appear explicitly but is taken to be determined by the gas law, Eq. (1.10). At mechanical equilibrium $\vec{v}=0, \dot{\rho}=0$ and $\lambda-g z=\hat{a} \gamma \rho^{1 / n}$, hence

$$
\rho=\left(\frac{\lambda-g z}{\hat{a} \gamma}\right)^{n}
$$

Since the density must be positive one does not fix the volume but assumes that the atmosphere ends at the point $z_{1}=\lambda / g$. Then

$$
M=\mathcal{A}\left(\frac{g}{\hat{a} \gamma}\right)^{n} \int_{z_{0}}^{z_{1}}\left(z_{1}-z\right)^{n} d z=\frac{\mathcal{A} h}{n+1}\left(\frac{g h}{\hat{a} \gamma}\right)^{n} .
$$

This fixes $h$ and thus $z_{1}$ and $\lambda$. If the atmosphere is an ideal gas then the temperature varies with altitude according to

$$
\mathcal{R} T=p / \rho=\frac{\hat{a}}{n} \rho^{1 / n}=g \frac{z_{1}-z}{n+1}
$$

For air, with atomic weight $29, \mathcal{R}=2.87 \times 10^{6} \mathrm{erg} / \mathrm{gK}$ and $n=2.5$. At sea level, $g=980 \mathrm{~cm} / \mathrm{s}^{2}$, the density is $\rho=1.2 \times 10^{-3} \mathrm{~g} / \mathrm{cm}^{3}$, the pressure $p=1.013 \times 10^{6}$ dyn $/ \mathrm{cm}^{2}$. Thus $p / \rho=0.844 \times 10^{9} \mathrm{~cm}^{2} / \mathrm{s}^{2}, T=T_{0}=294 \mathrm{~K}, z_{1}=3.014 \times$ $10^{6} \mathrm{~cm} \approx 30 \mathrm{~km}$. and the dry lapse rate at low altitudes is $-T^{\prime}=294 / z_{1}=9.75 \mathrm{~K} / \mathrm{km}$.

The specific internal energy of this model is $n p / \rho=n \mathcal{R} T$, as it should be for an ideal gas. However, the temperature is not treated as an independent dynamical variable, instead it is fixed by the constraint (1.10). The theory developed so far is partially "on shell".

A variational, principle is incomplete without the specification of boundary conditions. The only boundary condition that we shall apply systematically is the conservation of mass, which controls the material flow at the boundary.

\section{Thermodynamics}

\subsection{The adiabatic lagrangian}

The action principle can be extended so as to treat the temperature as an independent dynamical variable.

Two kinds of additions can be made to the lagrangian (1.9) without spoiling the equations of motion that are essential to hydrodynamics. To preserve the equations of motion as well as the correct expression for the internal energy, we have been led to the lagrangian for an ideal gas,

$$
\mathcal{L}[\Phi, \rho, T]=\rho\left(\dot{\Phi}-\vec{v}^{2} / 2-\phi+\lambda\right)-\mathcal{R} T \rho \log \frac{k}{k_{0}}+\frac{a}{3} T^{4}
$$


with $k:=\rho / T^{n}$. The last term is the contribution of radiation, assumed as is usual to be that of a black body; the constant $a$ is the Stefan-Boltzmann constant, $a=7.56 \times 10^{-15} \mathrm{ergs} / \mathrm{cm}^{3} \mathrm{~K}^{4}$.

Variation of the velocity potential $\Phi$ gives the continuity equation as before. Variation of the density $\rho$ leads to

$$
\dot{\Phi}-\vec{v}^{2} / 2-\phi+\lambda=\mathcal{R} T\left(1+\log \frac{k}{k_{0}}\right) .
$$

Taking the gradient one gets

$$
\rho \frac{D}{D t} \vec{v}-\rho \operatorname{grad} \phi=-\operatorname{grad} p
$$

where $D / D t=\partial / \partial t+\vec{\nabla} \cdot \vec{v}$ is the total derivative time and

$$
p=\mathcal{R} T \rho+\frac{a}{3} T^{4}
$$

Variation with respect to $T$ gives

$$
\mathcal{R}\left(n-\log \frac{k}{k_{0}}\right) \rho+\frac{4 a}{3} T^{3}=0,
$$

With the neglect of the radiation term, or if $n=3$, this reduces to the polytropic equation of change, $k=$ constant.

For conditions as in the atmosphere of the Earth, $\log \left(k / k_{0}\right)-n \approx 10^{-10}$. When the emphasis is not on applications we shall use units such that $k_{0}=1$.

The on shell hamiltonian density, obtained from (2.1) with the help of (2.5), takes the form

$$
h=\rho \vec{v}^{2} / 2+\rho \phi+u, \quad u=n \mathcal{R} T \rho+a T^{4} .
$$

We suggest that, when $n \neq 3$, using the lagrangian (2.1) is preferable to the usual assumption $\beta:=p_{\text {gas }} / p_{\text {tot }}=$ constant, which is true only when $n=3$.

We have an action principle, with dynamical variables $\rho$ and $T$, that reproduces all of the equations that characterize the equilibrium configurations, including the contribution of the photon gas to the energy and the pressure, as well as the standard, hydrodynamical relations of an ideal gas.

\subsection{Virial theorem}

Both (2.4) and (2.6) are usually derived from considerations outside the proper domain of thermodynamics. We prefer an axiomatic foundation of thermodynamics that is complete in the sense that it does not need other input. As an example let us discuss the use of the virial theorem to make certain predictions concerning stability. 
The virial theorem was introduced into the present context by Kelvin. It is based on the scaling properties of the hamiltonian of a system of particles. If $H=K+V$, kinetic energy plus potential energy, then the lagrangian is $K-V$ and the equations of motion imply that, up to a time derivative,

$$
\frac{d}{d t}(\vec{r} \cdot \vec{p})=2 K-\vec{r} \cdot \vec{\nabla} V
$$

where $\vec{p}=m \vec{v}, K$ is the kinetic energy $m \vec{v}^{2} / 2$ and $V$ is the potential. If the potential is homogeneous of degree $n$ then

$$
\frac{d}{d t}(\vec{r} \cdot p)=2 K-n V
$$

If the particle goes through a cycle then the average of this quantity over the cycle is zero. In the case examined by Kelvin the potential is homogeneous of degree -1 , so that, in the case of periodic motion, when average is taken over a period, $V=-2 K$. This is used to prove stability, since the total energy is $E=K+V=-K$ is negative.

The application to continuous systems, developed by Clausius, is much more difficult. According to Chandrasekhar [3, pp. 49-51], who also quotes Poincaré, the internal energy is the kinetic energy associated with the microscopic motion of the molecules. It is assumed, usually without discussion, that the presence of gravitational forces do not affect the internal energy, and that the total energy is obtained by simply adding the gravitational potential energy to it. In the present approach we are led to the same conclusion, the energy is identified with the hamiltonian. But the theory does not authorize drawing on kinetic theory to formulate a virial theorem. In fact, some attempts to do so have been successful only after making various assumption and/or approximations. See for example [4].

There is; however, a virial theorem associated with a lagrangian of the type (2.1), that we abbreviate as

$$
\mathcal{L}=\rho\left(\dot{\Phi}-\vec{v}^{2} / 2\right)-\hat{V}
$$

(The potential $\hat{V}$ includes the gravitational field and the lagrange multiplier). Variation of $\Phi$ and of $\rho$ gives the equations of motion

$$
\dot{\Phi}=\vec{v}^{2} / 2+(d \hat{V} / d \rho), \quad \dot{\rho}=-\operatorname{div}(\rho \vec{v}),
$$

In accord with the boundary condition discussed at the end of Sect. 1.3 (the conservation of mass), we drop the boundary term $-\int \Phi \rho \vec{v} \cdot \vec{d} \sigma$ to get

$$
\int d^{3} x \frac{d}{d t}(\rho \Phi)=\int d^{3} x\left(\rho \frac{d \hat{V}}{d \rho}-\rho \vec{v}^{2} / 2\right) .
$$


If the system goes through a cycle then the average of this quantity over the cycle is zero,

$$
\int d x \rho \vec{v}^{2} / 2=\int d x \rho \frac{d \hat{V}}{d \rho} .
$$

In the case of the lagrangian (2.1), but neglecting the radiation term, we obtain,

$$
\int d x\left(\rho \vec{v}^{2} / 2+\rho(\phi-\lambda)+\mathcal{R} T \rho(1+\log k)\right)=0 .
$$

To draw conclusions from this result we adopt the strategy of Jacobi, noting that the total energy is

$$
E=\int d^{3} x\left(\rho\left(\vec{v}^{2} / 2+\phi / 2\right)+\mathcal{R} T \rho \log k\right)
$$

By replacing the gravitational potential $\phi$ by $\phi / 2$ we are specializing to the case that the interaction is between the particles, not with a fixed source. In this case the interaction energy is bilinear in the density and variation of $\rho$ introduces a factor of 2 . The equations of motion are always the same but the factor $1 / 2$ is needed in the action.

Combining the last two equations we obtain

$$
\gamma E=\int d^{3} x\left(\frac{1}{n} \rho \vec{v}^{2} / 2+\left(\frac{1}{n}-1\right) \rho \phi / 2+\lambda \rho\right) .
$$

The last two terms can be evaluated. The calculation was done first by Ritter (1870); it is in many textbooks, and it is repeated later in this paper. The first result,

$$
\int d^{3} x \lambda \rho=\lambda M=\lambda \phi(R)=-\frac{G M^{2}}{R}
$$

is obtained with the help of the boundary condition at the surface and elementary properties of the gravitational interaction. The derivation of the following formula,

$$
\int d^{3} x \rho \phi / 2=E_{g}=\frac{-3}{5-n} \frac{M^{2} G}{R} .
$$

makes use the known degree of homogeneity $(\gamma)$ of the heat term with respect to the density. It is important that the equation of motion was not used. The sum is

$$
\int d^{3} x\left(\left(\frac{1}{n}-1\right) \rho \phi / 2+\lambda \rho\right)=\frac{(n-3)(n+1)}{(5-n) n} \frac{G M^{2}}{R} .
$$

This shows that, when $n>3$, the potential energy near the point of equilibrium is positive, which allows the gas to diffuse; the configuration is unstable. If $n=3$ there is a stable solution with zero energy, and another solution for which the energy is 
not defined. It just walks away, like a free particle or a critically damped harmonic oscillator.

\section{Stability of some atmospheres}

In all the examples of fluctuations around a static solution that follow we consider only adiabatic fluctuations; in the isothermal case the temperature is fixed and in the polytropic case the fluctuations of $T$ are governed by $\delta \rho / \rho=n \delta T / T$, by fixing $k=\rho / T^{n}$. One has to ask if this limitation to adiabatic fluctuations is fully justified. To study this question it is necessary to introduce entropy and to consider the heat equation, as we have attempted to do in the first paper [10]. As pointed out by Emden [7], it is necessary to assume that the heat flow has zero divergence, and this is supported by the fact that the polytropic atmosphere has constant lapse rate.

\subsection{The isothermal column}

The equations of motion (1.7), with $V=\mathcal{R} T \rho \log \rho$ (see Eq.(1.12)) are

$$
\dot{\rho}+(\rho v)^{\prime}=0, \quad \dot{v}+\left(v^{2} / 2+\mathcal{R} T(1+\log \rho)\right)^{\prime}=0 .
$$

The prime indicates derivation with respect to $z$. We consider the space that is tangent to a static solution with density $\rho_{0}$. Setting $\rho=\rho_{0}+\delta \rho$ we have the following equations for the perturbation $\delta \rho$,

$$
\delta \dot{\rho}+\left(\rho_{0} v\right)^{\prime}, \quad \dot{v}+\mathcal{R} T\left(\delta \rho / \rho_{0}\right)^{\prime}=0,
$$

Thus

$$
\delta \ddot{\rho}=\mathcal{R} T\left(\rho_{0} \alpha^{\prime}\right)^{\prime}, \quad \alpha:=\delta \rho / \rho_{0} .
$$

For a harmonic mode with frequency $\omega$,

$$
-\omega^{2} \delta \rho=R T\left(\rho_{0} \alpha^{\prime}\right)^{\prime}
$$

and

$$
-\frac{\omega^{2}}{\mathcal{R} T} \int \alpha^{2} \rho_{0} d z=\int \alpha\left(\rho_{0} \alpha^{\prime}\right)^{\prime} d z=\left.\rho_{0} \alpha \alpha^{\prime}\right|_{0} ^{\infty}-\int \rho_{o} \alpha^{\prime 2} d z
$$

The configuration is stable if this implies that $\omega^{2}>0$, which will be the case if the boundary term vanishes. To justify any choice of boundary conditions we have only the conservation of mass, $\int \delta \rho d z=0$. This ensures that $\delta \rho$ fall off at infinity and we are left with $-\delta \rho(0) \alpha^{\prime}(0)$. 
We shall show that $\alpha^{\prime}(0)=0$. Equation (3.1) tells us that

$$
-\frac{\omega^{2}}{\mathcal{R} T} \alpha=\left(\rho_{0}^{\prime} / \rho_{0}\right) \alpha^{\prime}+\alpha^{\prime \prime}=-\frac{g}{\mathcal{R} T} \alpha^{\prime}+\alpha^{\prime \prime} .
$$

This is a linear differential equation with constant coefficients, with general solution

$$
\alpha(z)=A \mathrm{e}^{\mathrm{k}_{+} \mathrm{z}}+\mathrm{Be}^{\mathrm{k}_{-} \mathrm{z}}, \quad \mathrm{k}_{ \pm}=\frac{\mathrm{g}}{2 \mathcal{R} \mathrm{T}} \pm \sqrt{\left(\frac{\mathrm{g}}{2 \mathcal{R T}}\right)^{2}-\frac{\omega^{2}}{\mathcal{R T}}} .
$$

Since, up to an irrelevant constant factor, $\rho_{0}=\exp (-g z / \mathcal{R} T)$,

$$
\delta \rho=\rho_{0} \alpha=A \mathrm{e}^{\mathrm{a}_{+} \mathrm{z}}+\mathrm{Be}^{\mathrm{a}_{-} \mathrm{z}}, \quad \mathrm{a}_{ \pm}=-\frac{\mathrm{g}}{2 \mathcal{R T}} \pm \sqrt{\left(\frac{\mathrm{g}}{2 \mathcal{R T}}\right)^{2}-\frac{\omega^{2}}{\mathcal{R T}}} .
$$

In order that $\delta M=\mathcal{A} \int \delta \rho d z$ vanish we need for the coefficients $A, B$ to be both non zero, and convergence of the integral then requires that $\omega^{2}>0$. In this case

$$
\delta M=\int \delta \rho d z=-\frac{A}{a_{+}}-\frac{B}{a_{-}}=\frac{A}{k_{-}}+\frac{B}{k_{+}},
$$

the vanishing of which requires that $\alpha^{\prime}(0)=0$. Therefore, not only is the condition $\omega^{2}>0$ verified; it is also confirmed that the boundary condition $\alpha^{\prime}(0)=0$ is the only one possible. We have seen that this choice of boundary conditions is the one that ensures the conservation of mass.

The fluctuation $\delta \rho$ does not vanish at the lower end, but the velocity does, as is natural. In fact, we can reach the same conclusion more easily as follows. Conservation of the current implies that

$$
\left.\rho v\right|_{0} ^{\infty}=0
$$

Since, as we showed, the upper limit cannot give a contribution, the velocity must vanish at the lower limit.

In this model, and in the one examined next, stability it physically obvious. The virial theorem has no interesting application in either case.

\subsection{The polytropic column}

Let us leave the parameter $k=\rho / T^{n}$ free and fix the value of $n$. This conforms to the usual approach when the temperature is fixed by edict, but it is consistent with our formulation if $n=3$ only, or in the case that the effect of radiation is neglected. We study the stability to vertical perturbations.

The static solution of (2.2) is

$$
c T=\lambda-g z, \quad c:=\mathcal{R}(1+\log k) .
$$


A first order perturbation satisfies

$$
\dot{\Phi}+\delta \lambda=c \delta T, \text { thus } \dot{v}=-c \delta T^{\prime}
$$

The equation of continuity gives

$$
\dot{\rho}=-(v \rho)^{\prime}, \quad \ddot{\rho}=-(\dot{v} \rho)^{\prime} .
$$

Let $x=\lambda / g-z, 0<x<\lambda / g$ and let $f^{\prime}=d f / d x$ from now on. Solutions of the type $\delta T=\exp (i \omega t) f(x)$ satisfy the equation

$$
\left(x^{n} \delta T^{\prime}\right)^{\prime}+\frac{v^{2}}{x}\left(x^{n} \delta T\right)=0, \quad v^{2}=n \omega^{2} / g .
$$

The solution that is regular at the origin of $x$ (the top of the atmosphere) is

$$
\delta T={ }_{0} F_{1}\left(n,-v^{2} x\right) \mathrm{e}^{\mathrm{i} \omega \mathrm{t}} .
$$

(Another solution may be obtained by the transformation $n \rightarrow 2-n, x \rightarrow 1 / x$.) The generalized hypergeometric function is positive for positive argument and it oscillates around zero for negative argument.

From (3.3) one obtains

$$
v^{2} \int x^{n-1}(\delta T)^{2} d z=-\int \delta T\left(x^{n} \delta T^{\prime}\right)^{\prime}=-\left.x^{n} \delta T \delta T^{\prime}\right|_{0} ^{\lambda / g}+\int x^{n}\left(\delta T^{\prime}\right)^{2} .
$$

Boundary conditions. If we fix $\delta T=0$ at the bottom of the column the boundary term vanishes, provided that the upper limit makes no contribution, and in this case the mass would be preserved. There are solutions for $v^{2}>0$ only, oscillatory in time.

The natural boundary condition is the preservation of the mass, thus

$$
\delta M=\int \delta \rho d x=n k \int T^{n-1} \delta T d x=0 .
$$

This may happen for a discrete set of positive values of $v^{2}$. For negative values of $v^{2}$ the integrand is definite so that it can not happen. The calculation is valid only in the case $n=3$ (for all $n$ when the effect of radiation is neglected); this atmosphere is stable.

The problem can be converted to a standard boundary value problem by rescaling of the coordinate.

\subsection{The polytropic gas sphere: the hamiltonian}

Here we study the self gravitating polytropic gas. A correction is needed in the expression for the lagrangian, and we need to take care with respect to the definition of the gravitational potential. 
The gravitational energy of a system in mutual interaction is the following functional of the mass density $\rho$,

$$
E_{g}[\rho]=-\frac{G}{2} \int d^{3} x d^{3} x^{\prime} \frac{\rho(\vec{x}) \rho\left(\vec{x}^{\prime}\right)}{\left|\vec{x}-\vec{x}^{\prime}\right|}
$$

It vanishes in the limit that the gas is diffused over an infinite volume and it is negative for all other density profiles.

This potential energy contributes to the hamiltonian so that we have to include a term $-E_{g}[\rho]$ in the lagrangian. It contributes to the equation of motion that comes from variation of $\rho$ a term $-\rho \phi$, as in the other cases examined, but now

$$
\phi[\rho](\vec{x})=\phi(\vec{x})=-\int d^{3} x \frac{G \rho\left(\vec{x}^{\prime}\right)}{\left|\vec{x}-\vec{x}^{\prime}\right|} .
$$

Our text books show how to evaluate the potential in the case of a spherically symmetric distribution, when one writes $\rho(x) \rightarrow \rho(r), \phi(x) \rightarrow \phi(r)$, with

$$
\phi(r)=-G \int \frac{\rho\left(r^{\prime}\right)}{z} r^{\prime 2} \sin \theta^{\prime} d r^{\prime} d \theta^{\prime} d \phi^{\prime}, \quad z=r^{2}+r^{\prime 2}-2 r r^{\prime} \cos \theta^{\prime}, \quad z>0 .
$$

The trick is to replace $\left(r^{\prime}, \theta^{\prime}\right)$ by $\left(\mathrm{r}^{\prime}, \mathrm{z}\right)$ as independent variables of integration, using

$$
z d z=r r^{\prime} \sin \theta^{\prime} d \theta^{\prime}
$$

The integral becomes

$$
\phi(r)=-2 \pi G \int \rho\left(r^{\prime}\right) \frac{r^{\prime}}{r} d r^{\prime} d z=-2 \pi G\left(I_{+}+I_{-}\right),
$$

where $I_{+}$is the contribution from the region $r^{\prime}<r$ and $I_{-}$is the rest. Now

$$
\begin{aligned}
& I_{+}=\frac{1}{r} \int_{r-r^{\prime}}^{r+r^{\prime}} \rho\left(r^{\prime}\right) r^{\prime} d r^{\prime} d z=\frac{2}{r} \int_{0}^{r} \rho\left(r^{\prime}\right) r^{\prime 2} d r^{\prime}, \\
& I_{-}=\frac{1}{r} \int_{r^{\prime}-r}^{r+r^{\prime}} \rho\left(r^{\prime}\right) r^{\prime} d r^{\prime} d z=2 \int_{r}^{\infty} \rho\left(r^{\prime}\right) r^{\prime} d r^{\prime},
\end{aligned}
$$

It results from this that

$$
\phi^{\prime}(r)=4 \pi G \frac{1}{r^{2}} \int_{0}^{r} \rho\left(r^{\prime}\right) r^{\prime 2} d r^{\prime} .
$$


the mass contained within the distance $r$ from the center is

$$
\mathcal{M}(r)=\int_{r^{\prime}<r} \rho\left(x^{\prime}\right) d^{3} x^{\prime},
$$

and the result of the calculation is that

$$
\phi^{\prime}(r)=\frac{G \mathcal{M}}{r^{2}}, \quad \phi(R)=-\frac{G \mathcal{M}}{R} .
$$

The potential is negative, increasing from the origin to the boundary and on towards zero at infinity, since for $r>R, \phi(r)=-G \mathcal{M} / r$.

We shall need three important integral formulas, the first two are found by by integration by parts,

$$
\begin{array}{r}
E_{g}=-4 \pi \int_{0}^{R} \frac{1}{2} \rho \phi r^{2} d r=\frac{1}{2} \int \mathcal{M}^{\prime} \phi d r=\frac{1}{2} M \phi(R)-\frac{1}{2} \int \mathcal{M} \phi^{\prime} d r \\
-\frac{1}{2} \int \mathcal{M} \phi^{\prime} d r=-\frac{1}{2} \int \frac{G \mathcal{M}^{2}}{r^{2}} d r=\frac{1}{2} \int G \mathcal{M}^{2}\left(\frac{1}{r}\right)^{\prime} d r=\frac{1}{2} \frac{G M^{2}}{R}-\int \frac{G}{r} \mathcal{M} d \mathcal{M} .
\end{array}
$$

The third is obtained by Eddington [6] by using the polytropic relation between $T$ and $\rho$. We need the equation of motion,

$$
\lambda-\phi=c T, \quad c=\mathcal{R}\left(\log \frac{k}{k_{0}}+\frac{n}{3}\right),
$$

but only to extract the boundary conditions. Since $T(R)=0$ it tells us that $\lambda=\phi(R)$. This field, namely

$$
\psi:=\phi(R)-\phi
$$

is Eddington's gravitational potential. The polytropic relation is seen as relating $\psi$ and $\rho$ and the result, here expressed in terms of $\phi$, is

$$
\int \frac{G}{r} \mathcal{M} d \mathcal{M}=\frac{3}{n+1} \int \mathcal{M} \phi^{\prime} d r^{\prime} .
$$

Combining (2) and (3) we obtain the data that will allow us to determine the value of the hamiltonian,

$$
\int \mathcal{M} \phi^{\prime} d r=\frac{n+1}{5-n} \frac{G M^{3}}{R}, \quad \int \frac{G}{r} \mathcal{M} d \mathcal{M}=\frac{3}{5-n} \frac{G M^{2}}{R}
$$


and

$$
E_{g}=-\frac{3}{5-n} \frac{G M^{2}}{R}
$$

The hamiltonian density is

$$
h=\rho \vec{v}^{2} / 2+\frac{1}{2} \rho \phi+\mathcal{R} T \rho \log k+\frac{a}{3} T^{4},
$$

or, in view of (2.5),

$$
h=\frac{1}{2} \vec{v}^{2}+\frac{1}{2} \rho \phi+\frac{3 c}{4} T \rho, \quad c=\mathcal{R}\left(\frac{n}{3}+\log k\right),
$$

whence the hamiltonian (= total energy) is

$$
H=\int d^{3} x h=E_{g}+\int d^{3} x\left(\frac{1}{2} \vec{v}^{2}+\frac{3 c}{4} T \rho\right) .
$$

Finally, the equation of motion $\lambda-\phi=c T$ yields

$$
\int c T d^{3} x=\lambda M+2 E_{g}=\frac{n+1}{5-n} \frac{G M^{2}}{R}
$$

Hence (4.12) reduces, in the static case, to

$$
H=-\frac{3}{5-n} \frac{G M^{2}}{R}+\frac{3}{4} \frac{n+1}{5-n} \frac{G M^{2}}{R}=\frac{3}{4} \frac{n-3}{5-n} \frac{G M^{2}}{R} .
$$

The conclusion that is drawn from this formula is that the equilibrium configuration is stable only if $n<3$.

Remark It is useful to calculate the relative contribution of radiation to the total energy; this can be done exactly when $n=3$, using the equation of motion (2.5),

$$
\mathcal{R} k\left(\log \frac{k}{k_{0}}-3\right)=\frac{4 a}{3} .
$$

We already gave a numerical example: in the case of the Earthly atmosphere $\log \left(k / k_{o}\right)$ $-n \approx 10^{-10}$. In general, the internal energy, with radiation included, can be expressed on shell as

$$
u=n \mathcal{R} T \rho+\frac{a}{3} T^{4}=: u_{\mathrm{gas}}+u_{\mathrm{rad}} .
$$


and it is natural to ascribe the first term to the gas and the other to radiation. Defining the quantity $\beta$ as Eddington does, namely

$$
\beta=p_{\mathrm{gas}} /\left(p_{\mathrm{gas}}+p_{\mathrm{rad}}\right)=u_{\mathrm{gas}} /\left(u_{\mathrm{gas}}+u_{\mathrm{rad}}\right),
$$

we obtain

$$
\beta=\frac{\log \left(k / k_{0}\right)-3}{\log \left(k / k_{0}\right)+1},
$$

which in the numerical example is approximately $0.25 \times 10^{-10}$.

\subsection{Historical review}

According to Kelvin, the conclusion that $n=3$ is critical for stability was announced by Perry, but Perrys paper could not be found. The same result was obtained, in the same year, by Ritter [15] and Betty. Ritter's calculation has often been repeated, but the original is by far the most coherent; it is very close to the one that we have given above.

Ritter does not formulate a lagrangian or a hamiltonian dynamics. He defines the total energy as the sum of the internal energy and the gravitational energy and postulates that this quantity is conserved. He did not have a dynamical theory in which the total energy is a well defined functional of the dynamical variables, but it is not a surprise to find that such a dynamical theory exists.

Later writers are much less careful. Eddington repeats all of Ritter's calculations, including the derivation of (3.8) that we have attributed to Eddington because his calculation is more easily accessible. But Eddington does not make use of the expression for the thermodynamical internal energy; instead he appeals to the interpretation of the gas as a collection of particles with mutual gravitational interactions. His claim that the result applies when radiation is taken into account is valid only in the case that $n=3$, as the reader can easily verify. Some modern writers give Ritter's calculation the attention that it merits [17], but some others take shortcuts that make their conclusions far less compelling. Kippenhahn and Weigert [13] reach the conclusion that $n=3$ is critical on the basis on an expression that is simply defined to be the total energy. Chandrasekhar (1958) also discusses this problem.

The above calculation made use of the polytropic relation, so it is valid in two cases: for all $n$ if the Stefan-Boltzmann term is neglected, and in the case $n=3$ whether the Stefan-Boltzmann term is included or not. Eddington claims the result for all $n$, but if $n \neq 3$, under the condition that the radiation pressure is a fraction of the total pressure, fixed throughout the star, which is not verified in our model, and under the additional condition that the polytropic relation between temperature and density is valid, which is also not verified.

All this leaves unsettled the question of stability for the case that the radiation term is included. However, if there is a unique, critical value of $n$ then it must be $n=3$, since Ritter's calculation applies to this special case, even when the energy of radiation is taken into account. 


\section{The polytropic gas sphere: stability}

Here we shall investigate the stability of the polytropic sphere directly, by perturbation theory.

We use the lagrangian

$$
\mathcal{L}=\rho\left(\dot{\Phi}-\vec{v}^{2} / 2-\phi / 2+\lambda\right)-\mathcal{R} T \rho \log k+\frac{a}{3} T^{4}, \quad k:=\rho / T^{n}
$$

Variation with respect to $T$ gives

$$
\mathcal{R}(\log k-n)=\frac{4 a}{3} T^{3} / \rho .
$$

With $n=3$ this makes $k$ a constant, and $\log k=n$ when radiation is neglected. In the remainder of this section, we set, for all values of $n$,

$$
\rho=k T^{n}, \quad k \text { constant }
$$

This is the usual polytropic relation used by Eddington and others, but it is consistent with (4.1) only when $n=3$. The remaining dynamical equations are

$$
\begin{aligned}
& -\frac{D v}{D t}=\phi^{\prime}+c T^{\prime}, \quad \dot{\rho}+r^{-2}\left(r^{2} \rho v\right)^{\prime}=0, \\
& 4 \pi G \rho=r^{-2}\left(r^{2} \phi^{\prime}\right)^{\prime}, \quad \rho=k T^{n} .
\end{aligned}
$$

- The static solution. Eliminate $\phi$ by $\phi^{\prime}=-c T^{\prime}$ and change variables, setting $r=x / \alpha, \alpha$ constant, Poisson's equation becomes

$$
\frac{4 \pi G k}{c \alpha^{2}} x^{2} T^{n}+\left(x^{2} T^{\prime}\right)^{\prime}=0
$$

where the prime now stands for differentiation with respect to $x$. Set $f(x)=T(x) /$ $T(0)$ and $\alpha=\sqrt{4 \pi G / c T(0)}$ so that finally

$$
x^{2} f^{n}+\left(x^{2} f^{\prime}\right)^{\prime}=0, \quad f(0)=1, \quad f^{\prime}(0)=0 .
$$

The solution decreases monotoneously to zero at $x=X$, this point taken to be the surface of the star. At the outer limit $f(x) \propto X / x-1+o(X-x)^{n}$. The integration is done easily and accurately by Mathematica, especially so for integer values of $n$. The radii are, for $n=2: X=4.355, n=3: X=6.89685636197, n=4: X=14.9715$.

- For the fluctuations we assume harmonic time dependence, then the equations are

$$
\begin{aligned}
& -\omega^{2} r^{2} \delta \rho=\left(r^{2} \rho\left(\delta \phi^{\prime}+c \delta T^{\prime}\right)\right)^{\prime}, \quad \delta \rho=n k T^{n-1} \delta T, \\
& 4 \pi G r^{2} \delta \rho=\left(r^{2} \delta \phi^{\prime}\right)^{\prime} .
\end{aligned}
$$


Introduce the function $\delta \mathcal{M}=r^{2} \delta \phi^{\prime}$. Equations (4.3) and (4.4) then take the form

$$
-\frac{\omega^{2}}{4 \pi G} \delta \mathcal{M}=\rho \delta \mathcal{M}+r^{2} \rho c \delta T^{\prime}+\text { constant }
$$

where the constant can only be zero, and

$$
(4 \pi G) r^{2}\left(n k T^{n-1} \delta T\right)=\delta \mathcal{M}^{\prime},
$$

Elimination of $\delta T$ leads to

$$
-\frac{\omega^{2}}{4 \pi G} \delta \mathcal{M}=\rho \delta \mathcal{M}+\frac{c}{4 \pi G k n} r^{2} \rho\left(\frac{\delta \mathcal{M}^{\prime}}{x^{2} T^{n-1}}\right)^{\prime} .
$$

Changing the scale as before we get

$$
-v^{2} \delta \mathcal{M}=f^{n} \delta \mathcal{M}+\frac{1}{n} x^{2} f^{n}\left(\frac{\delta \mathcal{M}^{\prime}}{x^{2} f^{n-1}}\right)^{\prime}, \quad v^{2}=\frac{\omega^{2}}{4 \pi G k T^{n}(0)}
$$

The crucial point is the choice of the correct boundary conditions, at $x=0$ as well as the outer surface $(x=X)$. At the center the solutions take one of two forms, $1+C x^{2}+\cdots$, which is unphysical, or else $x^{3}+C x^{5}+\cdots$. Accordingly we set

$$
\delta \mathcal{M}(x)=x^{3} g(x), \quad g(0)=1, \quad g^{\prime}(0)=0 .
$$

The boundary conditions at the outer boundary are determined by the fact that the mass is conserved,

$$
\delta M=\delta \mathcal{M}(X)=0 .
$$

The equations then imply that the zero is of order $n$. With these boundary conditions (4.5) becomes a well defined Sturm-Liouville problem with an essentially self adjoint, second order differential operator.

Numerical calculations with the help of Mathematica are not difficult in the case of integer values of $n$. It is found that, when $n=2$ and for $n=3, \delta \mathcal{M}(X)$ is positive in the whole range, for all negative values of $v^{2}$ and for positive values below a limit $v_{0}^{2}$ that is about 0.06 for $n=2$ and compatible with 0 for $n=3$. The latter is the first, nodeless solution of a sequence of solutions that we have not determined in detail. The function falls to zero at the surface, where there is an $n$th order zero. Above this lowest value of $v^{2}$ is a discrete set of other values of $v^{2}$ at which the boundary condition is satisfied.

At the special value $n=3$ the 'ground state', the lowest value of $v^{2}$, has approached very close to zero.

Polytropes with $n=4$ are unstable. We have searched for harmonic solutions with negative values of $v^{2}$. The value $n=4$ was chosen because it is the only integer in the interesting range, and because Mathematica is much more managable in this case. 
(Accuracy is lost when non integral powers of negative numbers appear at the end point.) There seems to be a discrete, decaying nodeless mode with $v^{2}=-0.015796$, but a bifurcation at this point in parameter space makes the conclusion uncertain. We carried the calculation to 15 significant figures in $v^{2}$ but solutions do not converge towards a function that vanishes at the surface. To overcome this difficulty we reformulated the problem in terms of the variational calculus. The "solution" found for $v^{2}=-0.015796$, truncated near both ends, was used as a trial function, to show conclusively that the spectrum of $v^{2}$ extends this far. Among many papers on this topic we mention Cowling [5] and Ledoux [14].

\section{The case $n=3$}

This case marks the boundary between stable and unstable polytropes. The equations are conformally invariant and a time independent solution is found by an infinitesimal conformal (homology) transformation,

$$
\delta f=r f^{\prime}+f .
$$

This does not represent an instability, but a "flat direction", a perturbation from which the system does not spring back, nor does it run away. There must also be a second solution, linear in $t$, of the form

$$
\delta f=t(r f)^{\prime}, \quad \delta \rho=t\left(r \rho^{\prime}+3 \rho\right) .
$$

The equation of continuity becomes $r \rho^{\prime}+3 \rho+v \rho^{\prime}+r^{-2}\left(r^{2} v\right)^{\prime} \rho=0$, whence $v=-r$.

This linear perturbation is the first order approximation to the exact solution found by Goldreich and Weber [11], of the form

$$
f(r, t)=\frac{1}{a(t)} \tilde{f}(x), \quad x=r / a(t) .
$$

The continuity equation is solved by $v=\dot{a} x$; thus $\Phi=-(\dot{a} / a)\left(r^{2} / 2\right)$, and

$$
\dot{\phi}-\vec{v}^{2} / 2=-a \dot{a} x^{2} / 2=c T+\phi .
$$

This leads to

$$
\tilde{\phi}:=a(t) \phi \propto \tilde{f}+\kappa a^{2} \ddot{a} x^{2} / 6, \quad \kappa=3 k^{1 / 3} / c,
$$

and Poisson's equation becomes

$$
\tilde{f}^{3}+\frac{1}{x^{2}}\left(x^{2} \tilde{f}^{\prime}\right)^{\prime}=\frac{-\kappa}{x^{2}} a^{2} \ddot{a} x^{2} / 6=-\kappa a^{2} \ddot{a}=\lambda, \text { constant. }
$$


There is a first integral,

$$
\frac{\kappa}{2} \dot{a}^{2}-\lambda / a=C, \text { constant. }
$$

Rescaling of $t$ and $a$ reduces this to one of three cases

$$
\dot{a}=\sqrt{1+1 / a}, \quad \dot{a}=\sqrt{1-1 / a}, \quad \dot{a}=1 / \sqrt{a},
$$

but only the first is compatible with analyticity at $t=0$, thus

$$
t=\sqrt{a} \sqrt{1+a}-\operatorname{arcsinh} \sqrt{a} .
$$

Setting $a=1+b$ we find

$$
t=\sqrt{1 / 2}\left(b-b^{2} / 2\right)+o\left(b^{3}\right)
$$

The factor $a(t)$ is zero at a finite, negative value of $t$ and increases monotoneously to infinity, passing through 1 at $t=0$. We can of course reverse the direction of flow of $t$ to get collapse in the finite future.

Equation (4.5) was solved numerically [11]. The solution is similar to the solution of Emden's equation, just prolonged a little at the outer end, so long as $0<\lambda<$ 0.00654376 . For larger values of $\lambda$ the distribution does not reach zero and increases for large $r$. For similar studies of collapsing, isothermal spheres see Hunter [12] and references therein.

It is confirmed, therefore, that the polytrope with $n=3$ is not stable. Suitably perturbed, the star may expand or collapse, until the higher or lower density causes a change in the equation of state. Among may papers on collapse we may mention Arnett [1] and Van Riper [16].

Open Access This article is distributed under the terms of the Creative Commons Attribution Noncommercial License which permits any noncommercial use, distribution, and reproduction in any medium, provided the original author(s) and source are credited.

\section{References}

1. Arnett, W.D.: Neutrino trapping during gravitational collapse of stars. Astrophys. J. 218, 815-833 (1977)

2. Bernoulli, D.: Argentorat (1738)

3. Chandrasekhar, S.: An Introduction to Stellar Structure. University of Chicago Press, Chicago (1938)

4. Collins, G.W.II: The virial Theorem in Stellar Astrophysics. http://www.scribd.com/doc/7573482/ (2007)

5. Cowling, T.G.: M.N.R.A.S. 101, 367 (1941)

6. Eddington, A.S.: The Internal Constitution of Stars. Dover, New York (1926)

7. Emden, R.: Gaskugeln. Teubner, Berlin (1907)

8. Fetter, A.L., Walecka, J.D.: Theoretical Mechanics of Particles and Continua. McGraw-Hill, New York (1980)

9. Fronsdal, C.: Ideal Stars and General Relativity. Gen. Relativ. Gravit. (2007)

10. Fronsdal, C.: Heat and Gravitation. I. The Action Principle. arXiv:0812.4990 (2008) 
11. Goldreich, P., Weber, S.V.: Homologously collapsing stellar models. Astrophys. J. 238, 991-997 (1980)

12. Hunter, C.: The collapse of unstable isothermal stars. Astrophys. J. 218, 834-845 (1977). 255-260

13. Kippenhahn, R., Weigert, A.: Stellar Structure and Evolution. Springer, Berlin (1990)

14. Ledoux, P.: Astrophys. J. 102, 143 (1945)

15. Ritter, A.: Wiedemann Annalen 11332 (1870). One of a series of papers in Wiedemann Annalen, now Annalen der Physik. For a list see Chandrasekhar (1938). The volumes 5-20 in Wiedemann Annalen appear as the volumes 241-256 in Annalen der Physik

16. Van Riper, K.A.: Astrophys. J. 221, 304 (1978)

17. Weinberg, S.: Gravitation and Cosmology. Wiley, New York (1972) 